\title{
“Una propuesta inclusiva de la enseñanza de la ubicación y localización espacial para estudiantes de grado 3"1
}

\author{
"An inclusive approach to teaching the location and \\ spatial location for students from Grade 3" \\ “Uma abordagem inclusiva para ensinar a localização e \\ localização espacial para estudantes de grau 3"
}

Recibido: mayo de 2013

Aceptado: agosto de 2013
Emerson Santana Sánchez ${ }^{2}$

Ángela Yadira Sparza Montenegro ${ }^{3}$

Diego Guerrero Garay ${ }^{4}$

Cindy Lorena Salcedo Leguizamón ${ }^{5}$

\section{Resumen}

En este documento se presenta una propuesta inclusiva de la enseñanza de la ubicación y localización espacial para estudiantes de grado $3^{\circ}$ incluyendo estudiantes con discapacidad visual, en la cual se desea realizar a la población inicialmente una apropiación conceptual por medio de procesos de formación e instrucción y un desarrollo del trabajo propuesto. Esta propuesta inclusiva en torno al pensamiento espacial, específicamente la localización (sistemas de referencia), permitirá que los estudiantes reconozcan y puedan describir las características de los sistemas de referencia, para ubicarse y orientarse en el espacio.

Palabras clave: Aprendizaje; NEES; alumnos discapacitados; orientación espacial; localización; geometría en tres dimensiones.

\begin{abstract}
This paper presents a proposal inclusive teaching location and spatial location for 3rd grade students including students with visual impairment, in which the people want to make an appropriation initially conceptual processes through training and education and development of the proposed work. This inclusive approach to thinking about space, specifically the location (reference systems), allow students to recognize and to describe the characteristics of the reference systems to locate and orient themselves in space.
\end{abstract}

Keywords: Learning, NEES, students with disabilities, spatial orientation, location, geometry in three dimensions.

\footnotetext{
1 Artículo de Investigación

2 Universidad Distrital Francisco José de Caldas, Bogotá, Colombia. Contacto: santana-emerson@hotmail.com

3 Universidad Distrital Francisco José de Caldas, Bogotá, Colombia. Contacto: angela.sparza@gmail.com

4 Universidad Distrital Francisco José de Caldas, Bogotá, Colombia. Contacto: diegorapmusic@hotmail.com

5 Universidad Distrital Francisco José de Caldas, Bogotá, Colombia. Contacto: lorenitalalinda15@hotmail.com
} 


\section{Resumo}

Este trabalho apresenta uma proposta de ensino inclusivo localização e localização espacial para os alunos da 3 a série, incluindo alunos com deficiência visual, em que as pessoas querem fazer uma apropriação processos conceituais inicialmente através da formação e educação e desenvolvimento do trabalho proposto. Esta abordagem inclusiva para pensar sobre o espaço, especificamente o local (sistemas de referência), permitir que os alunos a reconhecer e descrever as características dos sistemas de referência para localizar e orientar-se no espaço.

Palavras-chave: Aprendizagem, NEES, os alunos com deficiência, orientação espacial, localização, geometria em três dimensões.

\section{Presentación del problema}

Desde la perspectiva educativa, Sánchez (2003) considera que la diversidad se orienta a la aceptación y valoración de cada uno de los alumnos, y principalmente a reconocer la capacidad que tiene la población con discapacidad visual para aprender desde sus diferencias y la diversidad social (entorno familiar, escolar, económico, etc.) Teniendo en cuenta las diferencias individuales, es posible ofrecer igualdad de oportunidades. Pues cada estudiante es único, lleva un ritmo de aprendizaje único, y su educación inicial es el momento apropiado para intervenir en su proceso, pero solo al intervenir con calidad podría esperarse resultados, la efectividad de las estrategias implementadas y dar cuenta de si este cambio es o no permanente (duradero).

Allí se presenta la necesidad de diseñar prácticas organizadas previamente desde enfoques didácticos y comunicativos, pero esto siendo conscientes que no solo los estudiantes con necesidades especiales son los que necesitan este tipo de formación especial, sino todos aquellos estudiantes que a lo largo de su proceso educativo requieren una mayor atención que otro conjunto de estudiantes, incluso si los estudiantes no son de la misma edad.

En este sentido, la presente propuesta busca responder: ¿De qué manera estructurar a través del uso de recursos didácticos, el reconocimiento y la descripción de características de la localización (sistemas de referencia) para ubicarse y orientarse estudiantes limitados visualmente de grado $3^{\circ}$ en el espacio?. De esta manera la temática de recursos didácticos se aborda desde lo que propone Juan Godino cuando menciona la importancia del uso de material manipulativo tangible que permite contextualizar situaciones y facilitar el aprendizaje en los estudiantes.

\section{Marco de referencia conceptual}

La ubicación espacial es definitivamente una parte importante de la cultura del ser humano, en la antigüedad lograban ubicarse en el espacio ya fuera por la salida o la ponencia del sol o por las marcas que dejaban las estrellas, esto es solo una muestra de la necesidad de vivir cotidianamente conociendo al menos referentes espaciales, puntos cardinales, puntos de referencia, entre otros.

En la actualidad, actividades tan variadas como lo son buscar una dirección, tratar de encontrar algo perdido, llegar a un lugar desconocido, ubicar personas a nuestro alrededor, ubicarnos geográficamente, dar indicaciones a otra persona para que encuentre determinado lugar, ubicarnos mediante un mapa, ubicarnos mediante una brújula, entre otros, se sirven del conocimiento de nociones espaciales. En este sentido Bara (1975) citado por Fernández (2003) sostiene que..."el niño entiende el espacio en referencia a su propio cuerpo, de tal forma que cuando ubica su cuerpo en una superficie donde hay más personas u objetos, el niño desde su perspectiva de punto central, va organizando el espacio personal y el social y lo va haciendo en la medida que va conociendo sus posibilidades corporales".

Ahora para contribuir a que los alumnos superen las dificultades y/o vacíos que presentan en cuanto al manejo de las relaciones espaciales, debido a que las habilidades mentales que les permiten su manejo 
no se encuentran aun desarrolladas, se propone fortalecerlas mediante el uso de una progresión que propone Vayer (1974) citado por Arnaiz (1998), considerada como las vertientes que permiten conducir al niño al reconocimiento del espacio que ocupa su cuerpo y dentro del cual es capaz de orientarse, entre ellas:

- Diferenciación del yo corporal-mundo externo.

- Diferenciación de los segmentos corporales.

- Diferenciación de las relaciones espaciales con base en la referencia del propio yo corporal.

- Diferenciación de las relaciones espaciales con respecto al otro.

- Diferenciación de las relaciones espaciales con respecto a objetos entre sí.

- El paso de las relaciones en el espacio real a su representación en el espacio gráfico.

El uso de dicha progresión proporcionará a los alumnos desarrollar nuevos aprendizajes, adquirir nuevas nociones, manejar un nivel específico de vocabulario, permitiéndoles adquirir relaciones espaciales en todos sus aspectos.

De acuerdo a lo anterior la presente propuesta, pretende vincular a la población con discapacidad visual en el trabajo relacionado con el pensamiento espacial, enfatizando en el objeto matemático de la localización (sistemas de referencia), como menciona SED (2007) la localización tiene que ver..."con la capacidad de dar cuenta de la posición relativa de los objetos y para el cual el estudiante puede utilizar el cuerpo como sistema de referencia para ubicarse y orientarse en el espacio".

\section{Metodología}

La secuencia de actividades es diseñada siguiendo específicamente las fases de aprendizaje del modelo educativo de Van Hiele, donde se consideran las experiencias en la enseñanza-aprendizaje como la posibilidad de alcanzar niveles de razonamientos completos, sin olvidar que la misión de la educación matemática escolar es proporcionar experiencias adicionales muy bien estructuradas, para que sean lo más útiles posibles.

A lo largo de las fases, se debe procurar inicialmente que los alumnos adquieran comprensivamente los conocimientos básicos necesarios (nuevas nociones, relaciones, vocabularios) con los que tendrán que trabajar, para después centrar su actividad, en aprender a utilizarlos y relacionarlos. A continuación se revela la intención de forma general de cada una de las fases, atendiendo al objeto matemático que está en juego:

Finalmente brindada y trabajada la apropiación conceptual sobre localización y sistemas de referencia, los estudiantes están en la capacidad de dar cuenta de ello, construyendo un plano y posteriormente una maqueta como recurso para orientarse, fruto de la exploración concreta de un entorno real, así como la interpretación y representación gráfica y tangible, según las características significativas del mismo. Todo ello será posible a través de la formación e instrucción para cada una de las fases.

\section{Análisis de datos}

Esta categorización se realiza teniendo en cuenta la progresión mencionada por: Arnaiz, P (1998) (ver marco teórico), en cada elemento de la progresión se categorizan los estudiantes según los objetivos y actividades propuestas en las fases en donde se propuso desarrollar la progresión:

\section{Conclusiones}

- En la actualidad, actividades tan variadas como lo son buscar una dirección, tratar de encontrar algo perdido, llegar a un lugar desconocido, ubicar personas a nuestro alrededor, ubicarnos geográficamente, dar indicaciones a otra persona para que encuentre determinado lugar, ubicarnos mediante un mapa, ubicarnos mediante una brújula, entre otros, se sirven del conocimiento de nociones espaciales. 
Tabla 1

\begin{tabular}{|c|l|}
\hline FASE & \multicolumn{1}{|c|}{ INTENCIÓN } \\
\hline Información & $\begin{array}{l}\text { Identificar los conocimientos previos de los estudiantes sobre loca- } \\
\text { lización y sistemas de referencia (lateralidad, expresión corporal, } \\
\text { movilidad en el espacio, puntos cardinales, entre otros). }\end{array}$ \\
\hline Orientación dirigida y \\
explicitación & $\begin{array}{l}\text { Permitir (con la gestión del profesor) que los estudiantes exploren } \\
\text { el tema de estudio de localización (sistemas de referencia) por } \\
\text { medio de una progresión (remitirse a marco teórico) }\end{array}$ \\
\hline Orientación libre & $\begin{array}{l}\text { Relacionar los objetos de estudio trabajados hasta el momento (las } \\
\text { tres fases anteriores) con nuevas experiencias para que los estu- } \\
\text { diantes profundicen en la construcción de nociones de localización } \\
\text { y sistemas de referencia. }\end{array}$ \\
\hline
\end{tabular}

Fuente: Elaboración propia

- En general en las instituciones educativas se trabaja la localización espacial desde muy pequeños, pues los niños simplemente al nacer, se le observan movimientos inconscientes y reflejos, sin querer decir que ellos se orienten y tengan conciencia de su propio cuerpo en el espacio, de allí la necesidad de la intervención de la pedagogía para que estos conocimientos se estructuren.

- Los estudiantes con discapacidad visual necesitan al igual que los docentes, ese sentido de pertenencia dado por la vivencia cotidiana, desde el entorno externo social se debe favorecer e incentivar ese sentimiento, una integración efectiva demanda un ambiente que proporcione seguridad y estabilidad, que es favorecida por acciones en donde se respeten las diferencias, en donde la diversidad sea el punto de unión y no de desencuentro.

\section{Referencias}

Arnaiz, P. (1998). Educación infantil y deficiencia visual. Madrid. Ed. CCS.

Fernández, J. (2003). Teoría y práctica psicomotora de la orientación y localización espacial. Málaga, España. Ed. Búsqueda.

Sánchez, P. (2003). Educación inclusiva: Una escuela para todos. El reto de educar en y para la diversidad. Málaga, España. Ediciones Aljibe. 


\section{Anexos}

\begin{tabular}{|c|c|c|c|c|}
\hline CATEGORÍA & SUBCATEGORIAS & & & \\
\hline $\begin{array}{l}\text { Diferenciación del yo } \\
\text { corporal-mundo ex- } \\
\text { terno }\end{array}$ & $\begin{array}{l}\text { 1.1: Utiliza e identifica } \\
\text { las partes de su cuerpo } \\
\text { como primer sistema } \\
\text { de referencia. }\end{array}$ & $\begin{array}{l}\text { 1.2: Utiliza e identifica } \\
\text { las partes de su cuerpo } \\
\text { pero se confunde en } \\
\text { decir hacia donde es } \\
\text { la derecha o izquierda. }\end{array}$ & $\begin{array}{l}\text { 1.3: Utiliza e identi- } \\
\text { fica las partes de su } \\
\text { cuerpo pero se con- } \\
\text { funde en decir hacia } \\
\text { donde es arriba o } \\
\text { abajo. }\end{array}$ & $\begin{array}{l}\text { 1.4: Utiliza e iden- } \\
\text { tifica las partes de } \\
\text { su cuerpo pero se } \\
\text { confunde en decir } \\
\text { hacia donde es atrás } \\
\text { o adelante. }\end{array}$ \\
\hline $\begin{array}{l}\text { Diferenciación de } \\
\text { los segmentos corpo- } \\
\text { rales: brazos (arri- } \\
\text { ba)-piernas (abajo), } \\
\text { causada por la per- } \\
\text { cepción propiocepti- } \\
\text { va y exteroceptiva de } \\
\text { los movimientos. }\end{array}$ & $\begin{array}{l}\text { 2.1 Identifica que los } \\
\text { brazos están en la } \\
\text { parte de arriba y las } \\
\text { piernas en la parte de } \\
\text { abajo y también cuál } \\
\text { es su brazo y/o pierna } \\
\text { derecho (a) y cuál es } \\
\text { su brazo y/o pierna iz- } \\
\text { quierdo (a). }\end{array}$ & $\begin{array}{l}\text { 2.2: Identifica cuál es } \\
\text { su brazo y/o pierna } \\
\text { derecho (a) y cuál es } \\
\text { su brazo y/o pierna iz- } \\
\text { quierdo (a). }\end{array}$ & $\begin{array}{l}\text { 2.3: Identifica cuál } \\
\text { es su brazo y/o pier- } \\
\text { na derecho (a) y cuál } \\
\text { es su brazo y/o pier- } \\
\text { na izquierdo (a) pero } \\
\text { en ocasiones presen- } \\
\text { ta confusión. }\end{array}$ & $\begin{array}{l}\text { 2.4: No Identifica } \\
\text { que los brazos están } \\
\text { en la parte de arri- } \\
\text { ba y las piernas en } \\
\text { la parte de abajo y } \\
\text { tampoco reconoce } \\
\text { cual es su brazo y/o } \\
\text { pierna derecho (a) } \\
\text { y cuál es su brazo } \\
\text { y/o pierna izquier- } \\
\text { do (a). }\end{array}$ \\
\hline $\begin{array}{l}\text { Diferenciación de las } \\
\text { relaciones espaciales } \\
\text { en base a la referen- } \\
\text { cia del propio yo cor- } \\
\text { poral }\end{array}$ & $\begin{array}{l}\text { 3.1: Identifica si un } \\
\text { objeto se encuentra } \\
\text { lejos o cerca de su pro- } \\
\text { pio cuerpo. }\end{array}$ & $\begin{array}{l}\text { 3.2: Identifica si un } \\
\text { objeto se encuentra } \\
\text { detrás o adelante de su } \\
\text { propio cuerpo. }\end{array}$ & $\begin{array}{l}\text { 3.3: Identifica si un } \\
\text { elemento se encuen- } \\
\text { tra arriba o debajo de } \\
\text { un punto de referen- } \\
\text { cia (cara, ombligo, } \\
\text { rodillas). }\end{array}$ & $\begin{array}{l}\text { 3.4: Presenta difi- } \\
\text { cultad al momento } \\
\text { de identificar si un } \\
\text { elemento se en- } \\
\text { cuentra: cerca-lejos } \\
\text { de su cuerpo, de- } \\
\text { trás-delante de su } \\
\text { cuerpo, arriba-de- } \\
\text { bajo de un punto de } \\
\text { orientación. }\end{array}$ \\
\hline $\begin{array}{l}\text { Diferenciación de las } \\
\text { relaciones espaciales } \\
\text { con respecto al otro }\end{array}$ & $\begin{array}{l}\text { 4.1: Reconoce cual es } \\
\text { la parte derecha e iz- } \\
\text { quierda de una perso- } \\
\text { na (o objeto) que están } \\
\text { cara a cara. }\end{array}$ & $\begin{array}{l}\text { 4.2: Reconoce sola- } \\
\text { mente la parte derecha } \\
\text { de una persona (o ob- } \\
\text { jeto) que están cara a } \\
\text { cara. }\end{array}$ & $\begin{array}{l}\text { 4.3: Reconoce so- } \\
\text { lamente la parte iz- } \\
\text { quierda de una per- } \\
\text { sona (o objeto) que } \\
\text { están cara a cara. }\end{array}$ & $\begin{array}{l}\text { 4.4: Se confunde en } \\
\text { reconocer cual es la } \\
\text { parte derecha e iz- } \\
\text { quierda de una per- } \\
\text { sona (o objeto) que } \\
\text { están cara a cara. }\end{array}$ \\
\hline $\begin{array}{l}\text { Diferenciación de las } \\
\text { relaciones espaciales } \\
\text { con respecto a objetos } \\
\text { entre si }\end{array}$ & $\begin{array}{l}\text { 5.1: Reconoce que } \\
\text { con la mano derecha } \\
\text { se señala el oriente } \\
\text { (por donde sale el sol) } \\
\text { al lado opuesto (con } \\
\text { la mano izquierda) el } \\
\text { occidente, al frente el } \\
\text { norte y atrás el sur. }\end{array}$ & $\begin{array}{l}\text { 5.2: Reconoce que con } \\
\text { la mano derecha se } \\
\text { señala el oriente (por } \\
\text { donde sale el sol) al } \\
\text { lado opuesto (con la } \\
\text { mano izquierda) el oc- } \\
\text { cidente, pero se con- } \\
\text { funde con que al frente } \\
\text { es el norte y atrás es el } \\
\text { sur. }\end{array}$ & $\begin{array}{l}\text { 5.3: Reconoce que } \\
\text { con la mano derecha } \\
\text { se señala el oriente } \\
\text { (por donde sale el } \\
\text { sol) al lado opues- } \\
\text { to (con la mano iz- } \\
\text { quierda) el occiden- } \\
\text { te, pero en ocasiones } \\
\text { se confunde. }\end{array}$ & $\begin{array}{l}\text { 5.4: Se le dificulta } \\
\text { reconocer que con } \\
\text { la mano derecha } \\
\text { se señala el oriente } \\
\text { (por donde sale el } \\
\text { sol) al lado opues- } \\
\text { to (con la mano iz- } \\
\text { quierda) el occiden- } \\
\text { te, al frente el norte } \\
\text { y atrás el sur. }\end{array}$ \\
\hline
\end{tabular}

Fuente: Elaboración propia 